\title{
Effect of an experimental adhesive resin containing multi-ion releasing fillers on direct pulp-capping
}

\author{
Satoki KAWASHIMA 1 , Koichi SHINKAI ${ }^{2}$ and Masaya SUZUKI ${ }^{2}$ \\ ${ }^{1}$ Advanced Operative Dentistry, The Nippon Dental University Graduate School of Life Dentistry at Niigata, 1-8 Hamaura-cho, Niigata 951-8580, \\ Japan \\ ${ }^{2}$ Department of Operative Dentistry, The Nippon Dental University School of Life Dentistry at Niigata, 1-8 Hamaura-cho, Niigata 951-8580, Japan \\ Corresponding author, Koichi SHINKAl; E-mail: shinkaik@ngt.ndu.ac.jp
}

\begin{abstract}
The purpose of this study was to evaluate pulpal healing and reparative dentin formation after 14 and 28 days in exposed rat pulp directly capped with an experimentally developed all-in-one adhesive containing surface reaction-type pre-reacted glass-ionomer (S-PRG) filler. The four experimental groups and the control group were compared using the Kruskal-Wallis test, followed by the Steel-Dwass post-hoc test to compare the histopathological score. The Mann-Whitney U test was used to compare the histopathological score at 14 and 28 days for each observation item. All experimental adhesives containing S-PRG fillers developed for direct pulp capping showed no pulpal inflammation. After 14 days, the experimental adhesives containing S-PRG fillers and the control group formed tertiary dentin around the exposed pulp. After 28 days, the experimental adhesives containing 13 and $27 \mathrm{wt} \%$ of S-PRG fillers formed dentin bridge equal to the control.
\end{abstract}

Keywords: Direct pulp capping, Multi-ion releasing filler, Filler contents, Adhesive resin

\section{INTRODUCTION}

Accidental pulp exposure occasionally occurs during removal of deep caries or preparation of abutment teeth in clinics and is managed using calcium hydroxide for direct pulp capping. It has been reported that this calcium hydroxide induces the formation of a necrotic layer on the superficial exposed pulp, causing neutralization in the inflammatory pulp ${ }^{1-4)}$. However, limitations of calcium hydroxide include dead space formation due to solubility and inadequate mechanical strength ${ }^{1-6)}$. Additionally, because calcium hydroxide does not adhere to dentin or overlaid resins, sealing of the exposed site may remain incomplete. This may result in increased risk of pulp infection over time. Previous studies have introduced various direct pulp-capping agents, such as hydroxyapatite ${ }^{7}$, tri-calcium phosphate (TCP) ${ }^{8}$, mineral trioxide aggregate (MTA $)^{7,9,10)}$, and emdogain (EMD) ${ }^{9}$. MTA, in particular, has been shown to promote complete dentin bridge formation without inducing inflammatory response in the pulp ${ }^{7,9,10)}$. However, it also has limitations, such as high cost and difficult in handling compared to other pulp capping materials. The ideal pulp capping material should provide bonding efficacy to dentin around the pulp exposure site and prevent bacterial penetration. Recently, adhesive resin systems have been used as a direct pulp capping material; thus, simplifying the process of composite resin restoration and direct pulp capping. Moreover, the hybrid layer formed on the surface of the exposed pulp may protect it from bacterial infection. Some previous studies have reported pulpal irritation caused by the chemical composition of composite resins ${ }^{11-14}$. However, it has been recently shown that the major cause of pulpal irritation after composite resin restoration is due to the bacterial infection or chemical irritation induced by microleakage between the cavity wall and restoration ${ }^{5,6}$. Therefore, there has been an increasing interest in direct pulp capping using various adhesive systems. Several studies have reported the effects of various adhesive systems as direct pulp capping agents on the wound healing of exposed pulp ${ }^{4-6,11-21}$. Our previous studies using rat or human pulp showed that complete dentin bridge formation was achieved by proper application of an adhesive system on the exposed pulp ${ }^{15-18)}$. However, this was significantly slower than that observed with calcium hydroxide application ${ }^{17}$. Recently, Shofu, using the new technology, developed a functional filler called surface reaction-type pre-reacted glass-ionomer (S-PRG) that has bioactive effects. These fillers release aluminum (Al), boron (B), fluoride (F), sodium (Na), silicon ( $\mathrm{Si}$ ), and strontium (Sr) ions ${ }^{22-28)}$. Si and $\mathrm{F}$ ions, in particular, are known as strong inducers of remineralization of the dentin matrix ${ }^{29-32}$. It is also well known that $\mathrm{B}$ and $\mathrm{Sr}$ have osteogenic potential ${ }^{33-38}$. Sr promotes differentiation of pre-osteoblasts into osteoblasts ${ }^{33-37)}$, and it inhibits osteoclast differentiation and activity ${ }^{37}$. $\mathrm{B}$ is a trace element found in the human body that plays an important role in many life processes, including bone growth and maintenance ${ }^{38}$. On the other hand, few studies have reported a pulpal response to direct pulp capping using adhesives containing S-PRG fillers. Therefore, we developed a new, all-in-one adhesive containing various concentrations of S-PRG filler as a reparative dentin-promoting agent for direct pulp capping. We speculated that higher concentrations of S-PRG filler would demonstrate superior reparative dentin formation compared with that with lower concentrations of S-PRG. The purpose of this study was to evaluate pulpal healing and reparative dentin 
formation of exposed rat pulp directly capped with the experimentally developed all-in-one adhesive containing S-PRG filler. The null hypothesis of this study was that the S-PRG filler content in the experimental adhesives would not affect pulpal healing and reparative dentin formation in the exposed rat pulp.

\section{MATERIALS AND METHODS}

\section{Experimental animals}

Six-week-old Sprague-Dawley male rats, approximately $180 \mathrm{~g}$ in weight, were fed with solid food (MF, Oriental Yeast, Tokyo, Japan) and water for 2 weeks in the cages of the breeding house affiliated with our university. The teeth used in this study were maxillary first molars. Six teeth were randomly assigned to each experimental group $(n=6)$. A total of 60 non-carious upper molars were treated using direct pulp capping when the rodents were 8-9 weeks old and weighed 300-400 g. This study was approved by the Animal Committee of The Nippon Dental University School of Life Dentistry at Niigata (receipt and permission number: 152).

\section{Experimental groups and observation terms}

The materials used in this study are presented in Table 1 and the experimental groups are shown in Table 2. The experimental adhesives used in this study were developed in collaboration with Shofu, Kyoto, Japan. They consisted of Bond A and Bond B, where Bond A contained different S-PRG filler and silica filler contents (\% content given in Table 2 ). The total filler content was $40 \%$. Group 1 consisted of $0 \mathrm{wt} \%$ S-PRG filler and 40 wt\% silica filler; group 2 consisted of 13 wt $\%$ S-PRG filler and 27 wt\% silica filler; group 3 consisted of 27 wt\% S-PRG filler and 13 wt\% silica filler; and group 4 consisted of $40 \mathrm{wt} \% \mathrm{~S}-\mathrm{PRG}$ filler and $0 \mathrm{wt} \%$ silica filler. In all experimental groups, the components of Bond B were same. Pro Root MTA (DENTSPLY Tulsa Dental Specialties, OK, USA) was used as the control. Two postoperative observation periods (14 and 28 days) were fixed, and the rats were sacrificed on these days after direct pulp capping to allow preparation of specimens for histopathological and immunohistochemical examination.

\section{Specimen preparation}

The rats were intra-peritoneally injected with M/M/B:

Table1 Materials used in the present study

\begin{tabular}{|c|c|c|c|}
\hline Materials & Composition & Lot no. & Manufacturer \\
\hline $\begin{array}{l}\text { Experimental } \\
\text { bonding agents }\end{array}$ & $\begin{array}{l}\text { Bond A: S-PRG filler, Silica filler, Water, Solvent, Initiator } \\
\text { Bond B: 4-AET, 4-AETA, 6-MHPA, 2-HEMA, Bis-GMA, } \\
\text { Solvent, Initiator }\end{array}$ & 121031 & Shofu \\
\hline $\begin{array}{l}\text { Fluoro Bond } \\
\text { Shake One }\end{array}$ & $\begin{array}{l}\text { Bond A: S-PRG filler, FASG filler, Water, Solvent, Initiator } \\
\text { Bond B: 4-AET, 4-AETA, 6-MHPA, 2-HEMA, } \\
\text { Bis-GMA, Solvent, Initiator }\end{array}$ & 111201 & Shofu \\
\hline $\begin{array}{l}\text { Beautifil Flow } \\
\text { Plus F03 (A3) }\end{array}$ & $\begin{array}{l}\text { Bis-GMA, TEGDMA, Reaction initiator, } \\
\text { Powdered glass, Coloring agent }\end{array}$ & 111217 & Shofu \\
\hline Pro Root MTA & $\begin{array}{l}\text { Calcium oxide, Silicon dioxide, Aluminum oxide, } \\
\text { Bismuth oxide, Purified water }\end{array}$ & 13102906 & $\begin{array}{l}\text { DENTSPLY Tulsa } \\
\text { Dental Specialties }\end{array}$ \\
\hline
\end{tabular}

S-PRG filler: Surface reaction type pre-reacted glass-ionomer filler $(3 \mu \mathrm{m})$, FASG filler: Fluoro aluminosilicate glass filler $(1.5$ $\mu \mathrm{m})$

4-AET: 4-Acryloxyethyltrimellitic acid, 4-AETA: 4-Acryloxyethyltrimellitic anhydride

6-MHPA: 6-Methacryloyloxyhexyl phosphonoacetate, 2-HEMA: 2-Hydroxyethyl methacrylate

Bis-GMA: 2,2-bis[p-(2'-hydroxy-3'-methacryloxypropoxy)phenyl]propane, TEGDMA: Triethylen glycoldimethacrylate

Table 2 Experimental adhesives used in the present study

\begin{tabular}{cl}
\hline Group & \multicolumn{1}{c}{ Adhesive system } \\
\hline Group 1 & Bond A (S-PRG: 0 wt\%, Silica: 40wt\%)+Bond B \\
Group 2 & Bond A (S-PRG: 13 wt\%, Silica: 27wt\%)+Bond B \\
Group 3 & Bond A (S-PRG: 27 wt\%, Silica: 13wt\%)+Bond B \\
Group 4 & Bond A (S-PRG: 40 wt\%, Silica: 0wt\%)+Bond B \\
Control & MTA+Fluoro Bond Shake One \\
\hline
\end{tabular}


0.15/2/2.5 comprising of medetomidine (Domitor ${ }^{\circledR}$, Nippon Zenyaku Kogyo, Fukushima, Japan), midazolam (Sandoz ${ }^{\circledR}$, Sandoz K.K., Tokyo, Japan), and butorphanol (Vetorphale $^{\circledR}$, Meiji Seika Pharma, Tokyo, Japan) at a dose of $0.15 \mathrm{mg} / \mathrm{kg}+2.0 \mathrm{mg} / \mathrm{kg}+2.5 \mathrm{mg} / \mathrm{kg}$ body weight/ rat, respectively. The rodents were fixed on the operating table and their mouths were kept open with a mouth gag. The teeth were cleaned with $3 \% \mathrm{H}_{2} \mathrm{O}_{2}\left(\mathrm{Oxydol}^{\circledR}\right.$, Lots \#412140, Yoshida Pharmaceutical, Tokyo, Japan), rinsed with normal saline, and disinfected with diluted iodine tincture. Bowl-shaped cavities were made using an FG \#MI-1R regular cut diamond point (Shofu) in a high-speed handpiece (Air turbine handpiece, Super Load 9000, Yoshida Dental Trade Distribution, Tokyo, Japan) on the mesial marginal ridge of the maxillary first molars, and then, a CA \#1/2 steel round bur (Hager \& Meisinger, Neuss, Germany) in a low speed handpiece (Micromotor handpiece, Micro-Mega, Yoshida Dental Trade Distribution) was used to expose the pulps under copious water spray. The prepared cavities were treated with 10\% NaClO gel (AD gel, Lot \#1T0023, Kuraray Noritake Dental, Tokyo, Japan) for 5 min to stop bleeding from the exposed pulp and allow disinfection. If the pulp continued to bleed after this treatment, the same treatment was repeated. This was followed by alternate irrigation with $3 \% \quad \mathrm{H}_{2} \mathrm{O}_{2}$ and a $6 \% \mathrm{NaClO}$ solution (Purelox ${ }^{\circledR}$, Oyalux, Tokyo, Japan) to remove dentin chips and $\mathrm{AD}$ gel. The cavity was then rinsed with copious quantities of normal saline and gently air dried. The experimental direct pulp capping adhesive resin system was applied to the cavities. In the control, the exposed pulp was directly capped with Pro Root MTA, and then, Fluoro Bond Shake One was applied to the cavities. After $10 \mathrm{~s}$, the cavities were air dried and irradiated for $10 \mathrm{~s}$ with a light curing unit (Candelux ${ }^{\circledR}$, J Morita, Tokyo, Japan). After bonding procedures, all the cavities were restored with a flowable resin composite (Beautifil Flow Plus F03 A3, Shofu) and photopolymerized with the lightcuring unit for $40 \mathrm{~s}$.

\section{Perfusion fixation}

The rats were sacrificed using an intra-peritoneal injection of an overdose of the anesthetic solution mentioned above. Each pulp was fixed by trasnscardial vital perfusion with $4 \%$ paraformaldehyde phosphate buffer solution (4\% PFA, $\mathrm{pH}$ 7.4). The maxillae containing the experimental teeth were carefully removed and immersed in $4 \% \mathrm{PFA}$ at $4^{\circ} \mathrm{C}$ overnight for further fixation.

\section{Tissue preparation and serial sectioning}

The specimens were then trimmed to remove excess tissue and decalcified with 10\% EDTA decalcifying solution ( $\mathrm{pH}$ 7.4) at room temperature for 4 weeks. Thereafter, resin composite was carefully removed from the cavity, and the specimens were rinsed with running water for $24 \mathrm{~h}$. They were dehydrated in ascending grades of ethanol, dealcoholized by xylene, and then embedded in paraffin. Serial sections of $6 \mu \mathrm{m}$ thickness were cut using a sliding microtome (Jung Histlide
2000R, Leica Microsystems Vertrieb, Wetzlar, Germany) and alternately stained with Mayer's hematoxylin-eosin staining (H\&E) and Gram bacterial staining. They were then immunohistochemically stained using the streptavidin biotin complex method (SAB). The stained sections were observed under a light microscope (Eclipse E 100, Nikon, Tokyo, Japan), and the following items were evaluated: pulp tissue disorganization (PTD), inflammatory cell infiltration (ICI), reparative dentin formation (RDF), and bacterial penetration (BP). Nestin staining and HSP25 staining were used for immunohistochemical staining.

\section{Observation items and evaluation criteria}

The findings were evaluated according to the following criteria $^{15)}$.

1. Pulp tissue disorganization (PTD)

1) Normal or almost normal tissue morphology (none).

2) Odontoblast layer disorganized but the deep part of the pulp was normal (mild).

3) Loss of general tissue morphology (moderate).

4) Necrosis in the coronal third or more of the pulp (severe).

2. Inflammatory cell infiltration (ICI)

1) Absence or presence of a few scattered inflammatory cells in the pulp (none).

2) Mild acute/chronic cell lesions (mild).

3) Moderate inflammatory cell lesions were observed as densely stained infiltrates of polymorphonuclear leucocytes, histiocytes, and lymphocytes in one-third or more of the coronal pulp and/or the mid-pulp (moderate).

4) Pulp necrosis due to a severe degree of infection or lack of tissue in half or more of the pulp (severe).

3. Reparative dentin formation (RDF)

1) No dentin bridge formation (none).

2) Initial dentin bridge formation extending to not more than one-half of the exposure site (initial).

3) Partial/incomplete dentin bridge formation extending to more than one-half of the exposure site but not completely closing the exposure site (partial).

4) Complete dentin bridge formation (complete).

4. Bacterial penetration (BP)

1) Absence of stained bacterial profiles in any of the sections (none).

2) Presence of stained bacterial profiles along the coronal or apical walls of the cavity (mild).

3) Presence of stained bacterial profiles within the cut dentinal tubules or axial wall of the cavity (moderate).

4) Presence of stained bacterial profiles within the dental pulp (severe). 


\section{Immunohistochemical staining}

In this study, the expression of nestin and HSP25 was investigated as an index or marker for comparing the difference in the recovery process induced by the various direct pulp capping methods. The sections were deparaffinized with xylene, hydrated in ascending grades of ethanol, and then rinsed briefly with tap water and phosphate buffered saline (PBS, pH 7.4). They were then incubated with primary rabbit antibodies, such as polyclonal anti-nestin (Lot \#BS-0008R, Bioss, USA) with a working dilution of $1: 400$ for $12 \mathrm{~h}$ at $4^{\circ} \mathrm{C}$ or polyclonal anti-HSP25 (Lot \#AB138366, Abcam, UK) with a working dilution of $1: 400$ for $12 \mathrm{~h}$ at $4^{\circ} \mathrm{C}$, and then rinsed with PBS three times for $5 \mathrm{~min}$. They were immunochemically stained with the enhanced polymer one-step staining (EPOS) methods, Simple Stain Rat MAX-PO (R) (Lot \#H1403, Nichirei Biosciences, Tokyo, Japan). The antibody localized antigen was then detected by peroxidase activation of 3,3'-diaminobenzidine, Histofine Simple Stain DAB (Lot \#415171, Nichirei Bioscience) for $10 \mathrm{~min}$. Finally, the sections were lightly counterstained with Mayer's hematoxylin. Negative control staining was performed by replacing the primary antibody with phosphate-buffered saline.

\section{Measurement of the diameters of exposed pulp area}

The diameter of the exposed areas was measured with a stereomicroscope (Measuring Microscope MM-40, Nikon) and the widest dimension was recorded as the pulp exposure size of the specimen.

\section{Acid buffering capacity test and measurement of released ions}

Each experimental adhesive Bond A was mixed with Bond B, followed by intensive air-blowing for $5 \mathrm{~s}$. Each mixture was poured into a metal mold (diameter: 15 mm, thickness: $1 \mathrm{~mm}$ ) with a thin glass plate backing. The other side of the metal mold was also covered with a thin glass plate, and the mixture was light-cured for 3 min from each side using a light curing unit (Solid lite V, Shofu). All adhesive disks were removed from the metal mold, pressed between two thick glass plates for $24 \mathrm{~h}$, and then both surfaces of the disks were slightly ground using a 600 grit paper with polishing machine (Lewel specimen polisher, Kasai, Yokohama, Japan) under water irrigation. Eight disks were made for the respective experimental groups and used as test specimens for an acid buffering capacity test and a measurement of released ions.

Each specimen of each experimental group was immersed in $5 \mathrm{~mL}$ of $37^{\circ} \mathrm{C}$ lactic acid solution adjusted to $\mathrm{pH} 4$ for acid buffering capacity test $(n=2)$. The $\mathrm{pH}$ of the solution was measured using a $\mathrm{pH}$ meter $(\mathrm{pH}$ METER LAQUA D-51, HORIBA, Kyoto, Japan) after 0, $1,2,3,4,8,16$, and $24 \mathrm{~h}$. In this experiment, lactic acid solution adjusted to $\mathrm{pH} 4$ without a specimen was used as control.

Each specimen of each experimental group was immersed in $5 \mathrm{~mL}$ distilled water for 2 weeks or 4 weeks $(n=6)$. For measurement of released $\mathrm{F}$ ion, an ionic strength adjuster (TISABIII, Orion Research, Japan) was added to the distilled water after the respective storage terms. The amount of $\mathrm{F}$ ion released was measured using a F ion electrode (Model 960BN, Orion Research) with a pH/ion meter (Model 720A, Orion Research). For measurement of released $\mathrm{Sr}, \mathrm{B}, \mathrm{Na}, \mathrm{Al}$, and Si ion, nitric acid (NACARAI TESQUE, Japan) was added to the distilled water after the respective storage terms. The quantities of the ions released were measured using an inductively coupled plasma atomic emission spectrometer (ICP) (ICP-8000, Shimadzu, Kyoto, Japan).

\section{Statistical analysis}

The diameter of the exposed pulp areas were statistically analyzed using one-way ANOVA, and the Tukey post$h o c$ test was used for differences between the groups during each observation period. The significance level was set at 0.05 (Microsoft Excel 2010 for Windows, SSRI, Tokyo, Japan). The data from the histopathological evaluation at each observation period was statistically analyzed using the Kruskal-Wallis test followed by the Steel-Dwass post-hoc test to compare the histopathological score between the five experimental groups (Microsoft Excel 2010 for windows, SSRI). The data from the histopathological evaluation of each observation was statistically analyzed using the MannWhitney $U$ test to compare the scores at 14 and 28 days post-surgery (Microsoft Excel 2010 for windows, SSRI). The data on the quantity of released ions after each storage term was analyzed using one-way ANOVA followed by the Tukey-HSD post hoc test (Microsoft Excel 2010 for windows, SSRI).

\section{RESULTS}

\section{Histopathological findings}

The results of the histopathological evaluation after 14 and 28 days post-surgery are shown in Figs. 1 and 2 , respectively. Representative histopathological and immunohistochemical images of some groups are shown in Figs. 3 and 4.

\section{After 14 days}

The Kruskal-Wallis test for data of PTD and ICI showed no significant differences between the groups $(p>0.05)$. On the other hand, the Kruskal-Wallis test for RDF showed a significant difference $(p=0.0329)$, and the Steel-Dwass post-hoc test showed a significant difference between group 1 and control $(p=0.0152)$. None of the specimens in all groups except one specimen in group 2 showed complete dentin bridge. The specimen of group 1 showed no reparative dentin formation (Fig. 3-a). The specimens of group 2 and control showed reparative dentin formation in the pulp tissue away from the exposed pulp surface (Figs. 3-b and f). Specimens of groups 3 and 4 showed thin reparative dentin formation on the exposed pulp surface (Figs. 3-c and -d).

Nestin staining showed a slight positive reaction just under the reparative dentin in some specimens of 

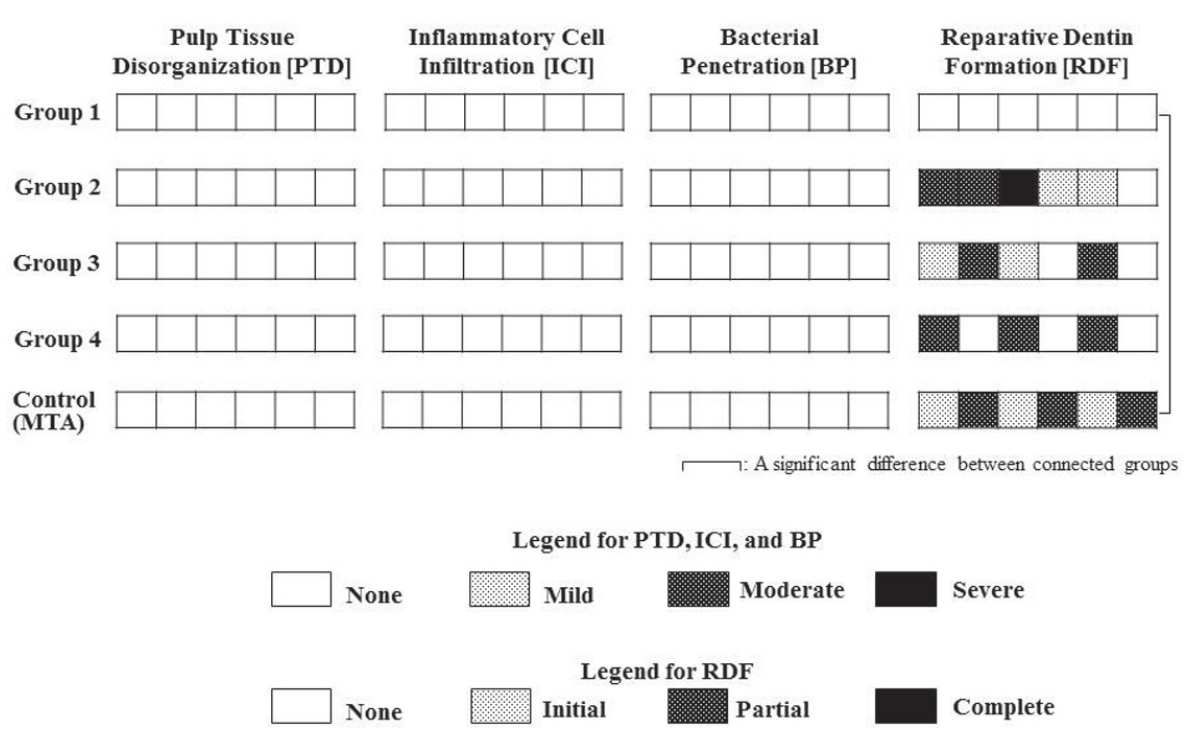

Fig. 1 Results of the histopathological evaluation (14 days).
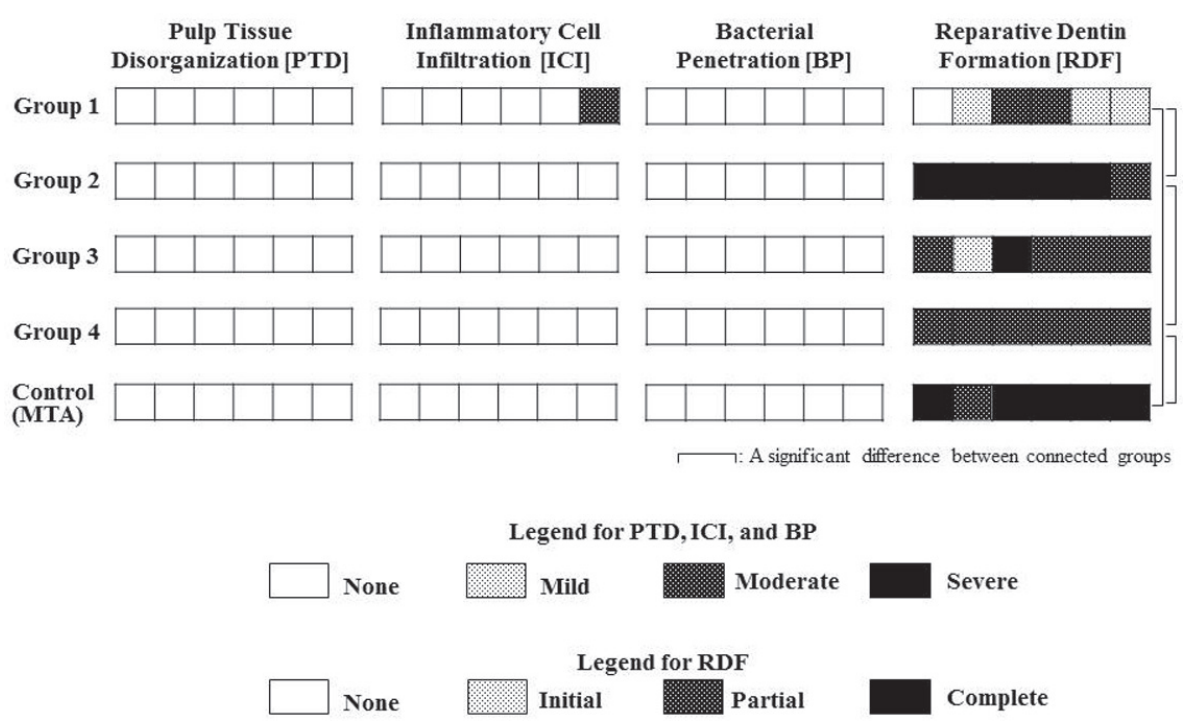

Fig. 2 Results of the histopathological evaluation (28 days).

the control after 14 days (Fig. 3-g). HSP25 immunoreactivity was observed under the reparative dentin and in pulp tissue slightly distant from the exposed pulp surface (Figs. 3-e and h).

\section{After 28 days}

The Kruskal-Wallis test for data of PTD and ICI showed no significant differences between the groups $(p>0.05)$. On the other hand, the Kruskal-Wallis test for data of the RDF showed a significant difference $(p=0.0006)$. The Steel-Dwass post-hoc test revealed a significant difference between group 1 and group $2(p=0.0338)$, group 1 and control ( $p=0.0338$ ), group 2 and group 4 $(p=0.0405)$, and group 4 and control $(p=0.0405)$. Moderate inflammatory cell infiltration was observed in group 1 , and reparative dentin formation was observed in all groups except group 1. Various types of reparative dentin were formed adjacent to the pulp exposure site. Groups 2 and 3 showed complete dentin bridge formation equal to the control; however, they were thinner than those observed in the control. In some specimens, reparative dentin or dentin bridge was formed at the middle and deep positions of pulp tissues (Figs. 4-b, c and d). Most specimens of the control showed complete dentin bridge formation. The dentin bridges were almost accompanied with odontoblastic layers beneath them (Figs. 4-c, $d$ and f). Some specimens showed involvement of the silica fillers in the pulp tissue (Figs. 4-a and b). 

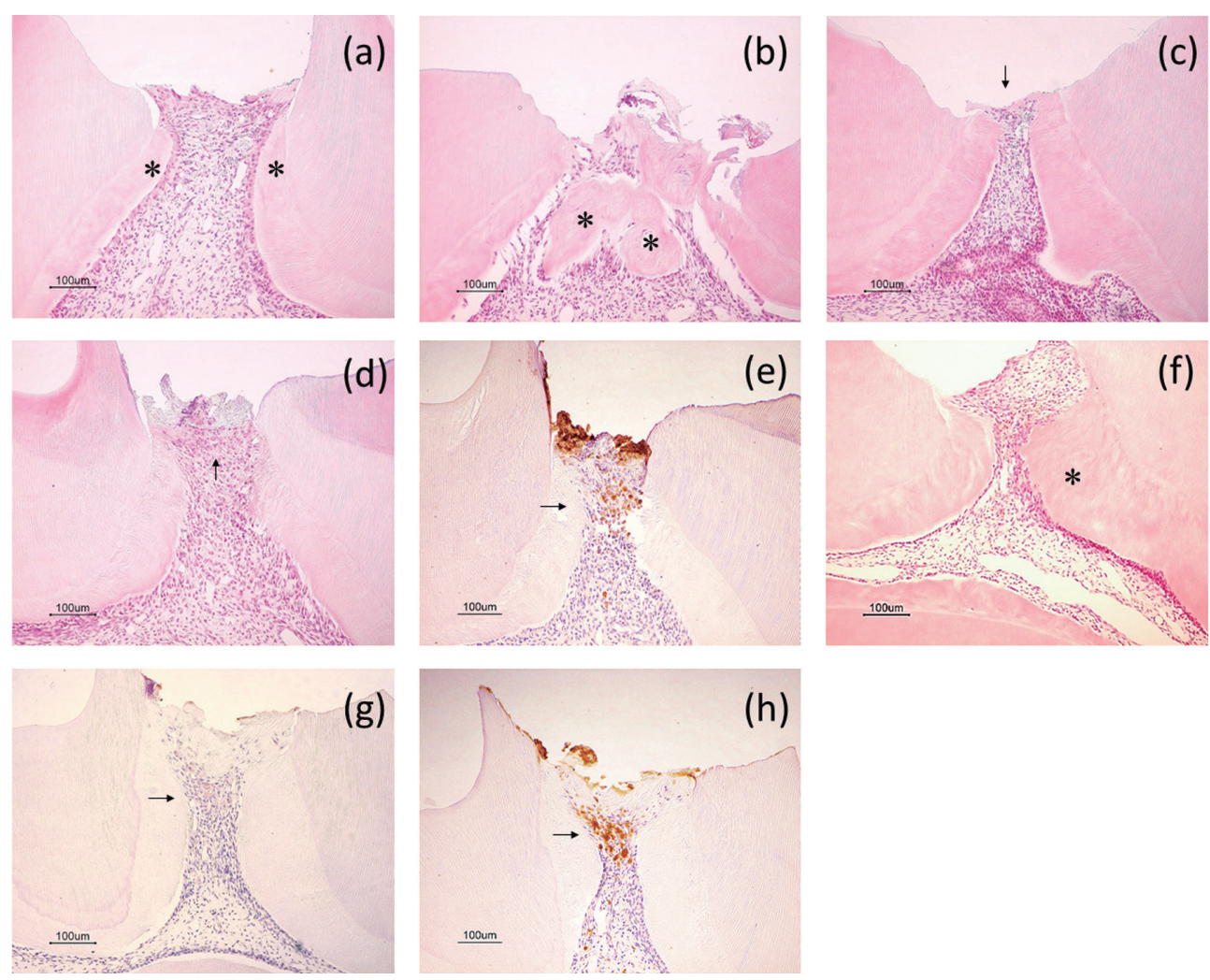

Fig. 3 Representative histologic images 14 days post-surgery.

(a) Group 1; Although reparative dentin (asterisk) was formed in the pulpal dentin wall at the periphery of the exposed site, there was no evidence of RDF at the exposed surface. PTD, ICI and BP: None, RDF: None (H\&E, 100×), (b) Group 2; Reparative dentin (asterisk) was observed at a position comparatively deeper than the site of pulpal exposure. PTD, ICI and BP: None, RDF: Partial (H\&E, 100×), (c) Group 3; Thin dentin bridge formation (arrow) was observed on the exposed pulp surface. PTD, ICI and BP: None, RDF: Partial (H\&E, 100×), (d) Group 4; Thin dentin bridge formation (arrow) was observed on the exposed pulp surface. PTD, ICI and BP: None, RDF: Partial (H\&E, 100×), (e) Group 4; Strong positive reaction by HSP25 staining (arrow) can be seen under the exposed pulp surface. (HSP25, 100×), (f) Control; A greater amount of reparative dentin (asterisk) was induced at the exposed site. PTD, ICI and BP: None, RDF: Partial (H\&E, 100×), (g) Control; Nestin staining (arrow) showed a slight positive reaction just under reparative dentin. (Nestin, 100×), (h) Control; A strong positive reaction by HSP25 staining (arrow) can be seen in the dentin bridge and reparative dentin (HSP25, 100×).

Mann-Whitney U test for data of PTD and ICI revealed no significant differences between 14 and 28 days $(p>0.05)$ in any experimental groups or control. The same test for data of the RDF showed significant differences between 14 and 28 days in group $1(p=0.0068)$, group 2 ( $p=0.0198)$, and control $(p=0.0049)$, but no significant differences were observed between 14 and 28 days in group 3 or group 4 ( $p>0.05)$.

After 28 days, no nestin or HSP25 immunoreactivity was observed in any group except one specimen each in groups 1 and 4 (Figs. 4-e and g).

The diameters of the pulp exposure area

The mean value \pm standard deviation of the pulp exposure size for each group ranged from $0.278 \pm 0.025 \mathrm{~mm}$ to $0.313 \pm 0.031 \mathrm{~mm}$. There were no significant differences in pulp exposure size between groups $(p>0.05)$.

\section{Acid buffering capacity test}

Figure 5 shows the changes in solution $\mathrm{pH}$ with the passage of time for each experimental group. The mean values of the solution $\mathrm{pH}$ in groups 2 , 3, and 4 were higher than that of the control group at all measuring times. Group 4 showed the highest acid buffering capacity and achieved neutral $\mathrm{pH}$ value after $16 \mathrm{~h}$. On the other hand, the solution $\mathrm{pH}$ of group 1 was lower than that of control at all measuring times. 

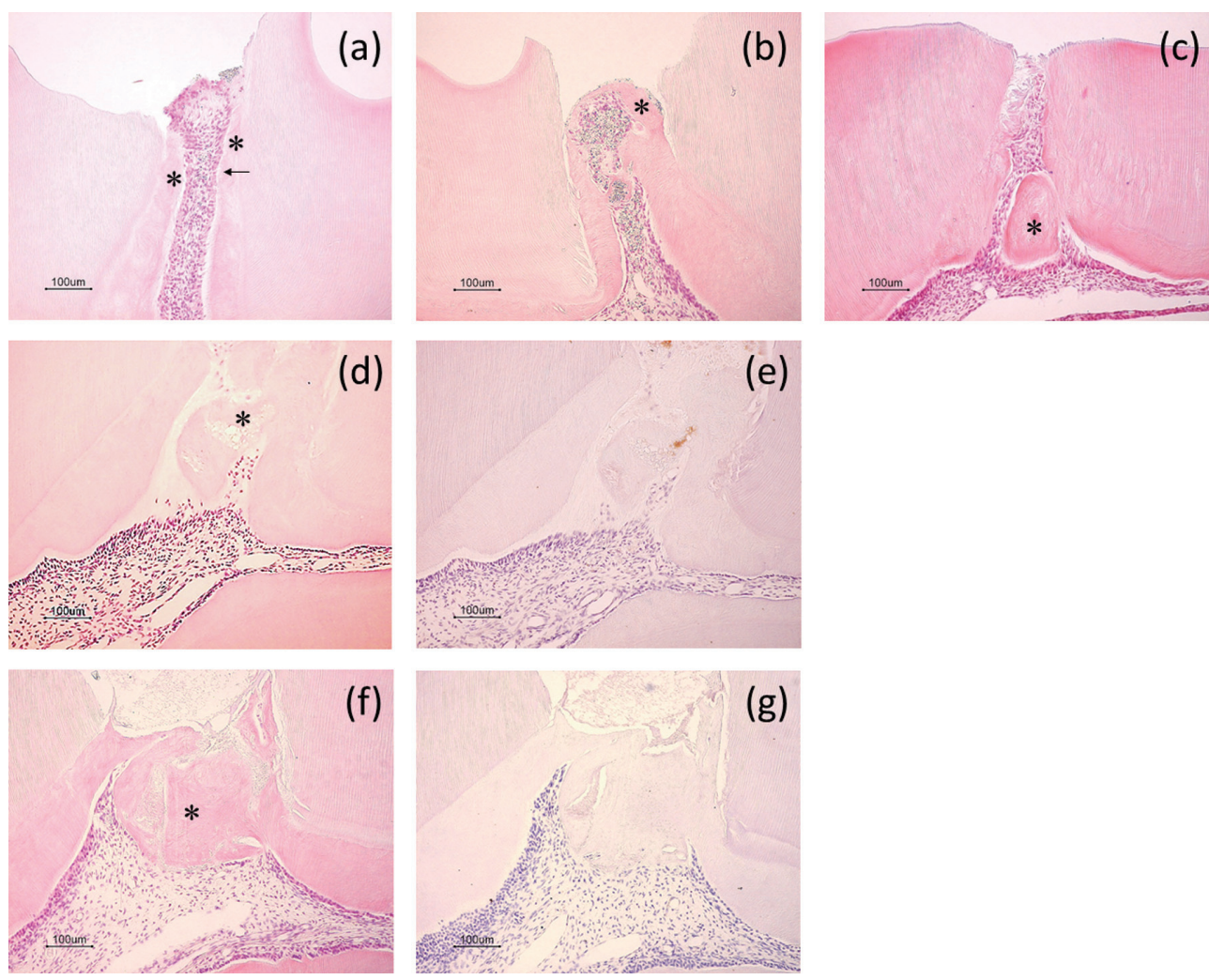

Fig. 4 Representative histologic images 28 days post-surgery.

(a) Group 1; Slight reparative dentin formation (asterisk) was observed at the surface of the pulp exposure site 28 days post-surgery. Involvement of silica fillers (arrow) was observed in the pulp tissue. PTD, ICI and BP: None, RDF: Initial (H\&E, 100×), (b) Group 2; The area of exposed pulp is completely surrounded by dentin bridge formation (asterisk). Involvement of silica fillers was observed in the pulp tissue. PTD, ICI and BP: None, RDF: Complete (H\&E, 100×), (c) Group 3; Dentin bridge formation (asterisk) was observed at the exposed surface, but this was incomplete. PTD, ICI and BP: None, RDF: Partial (H\&E, 100×), (d) Group 4; Dentin bridge (asterisk) was formed at deep position of pulp tissues. PTD, ICI and BP: None, RDF: Partial (H\&E, 100×), (e) Group 4; HSP25 staining showed no reaction in the dentin bridge. (HSP25, 100×), (f) Control; Thick, complete dentin bridge (asterisk) was observed at a position deeper than the pulpal exposed site. PTD, ICI and BP: None, RDF: Complete (H\&E, 100×), (g) Control; Nestin staining showed no reaction in the dentin bridge (Nestin, 100×).

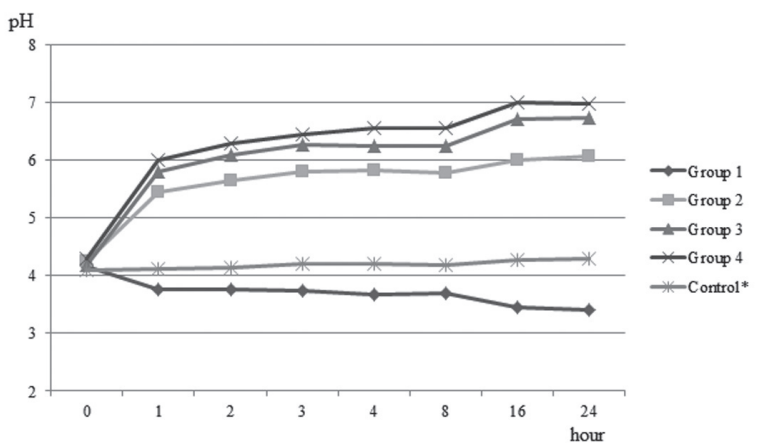

* Lactic acid solution adjusted to $\mathrm{pH} 4$ without a specimen was used as control.

Fig. $5 \mathrm{pH}$ changes of lactic acid solution mixed with S-PRG filler.

\section{Measurement of released ions}

Table 3 shows the mean quantity of respective ions released from the experimental adhesives during each storage term. Groups 3 and 4 released significantly greater amounts of $\mathrm{Na}, \mathrm{B}, \mathrm{Sr}$, and $\mathrm{F}$ ions during both storage terms compared to group 2. On the other hand, the mean amount of Si ion released from the experimental adhesive used in group 2 was significantly more than that used in groups 3 and 4 . There were no significant differences between group 3 and group 4 in the mean amount of $\mathrm{Na}, \mathrm{Sr}$, and $\mathrm{F}$ ions released after both storage terms. The experimental adhesive used in group 1 did not release $\mathrm{B}, \mathrm{Sr}$, and $\mathrm{F}$ ions. The amount of $\mathrm{Si}$ and $\mathrm{Sr}$ ions released after 28 days was significantly greater than that after 14 days in all experimental groups. 
Table 3 Amount of ion released (ppm) from S-PRG filler into distilled water at 14 and 28 days

\begin{tabular}{|c|c|c|c|c|c|c|c|c|c|c|c|c|}
\hline \multirow{2}{*}{$\begin{array}{l}\text { Ions } \\
\text { Days }\end{array}$} & \multicolumn{2}{|c|}{$\mathrm{Na}$} & \multicolumn{2}{|c|}{ B } & \multicolumn{2}{|c|}{$\mathrm{Al}$} & \multicolumn{2}{|c|}{$\mathrm{Si}$} & \multicolumn{2}{|c|}{$\mathrm{Sr}$} & \multicolumn{2}{|c|}{$\mathrm{F}$} \\
\hline & 14 & 28 & 14 & 28 & 14 & 28 & 14 & 28 & 14 & 28 & 14 & 28 \\
\hline Group 1 & $\begin{array}{c}197.99^{\mathrm{abA}} \\
(6.70)\end{array}$ & $\begin{array}{c}195.97^{\mathrm{cA}} \\
(26.60)\end{array}$ & $\begin{array}{c}0.00 \\
(0.00)\end{array}$ & $\begin{array}{c}0.00 \\
(0.00)\end{array}$ & $\begin{array}{l}2.26^{\mathrm{F}} \\
(0.60)\end{array}$ & $\begin{array}{l}3.76^{\mathrm{F}} \\
(0.38)\end{array}$ & $\begin{array}{c}7.68 \\
(0.99)\end{array}$ & $\begin{array}{l}13.12 \\
(2.42)\end{array}$ & $\begin{array}{c}0.00 \\
(0.00)\end{array}$ & $\begin{array}{c}0.00 \\
(0.00)\end{array}$ & $\begin{array}{c}0.00 \\
(0.00)\end{array}$ & $\begin{array}{c}0.00 \\
(0.00)\end{array}$ \\
\hline Group 2 & $\begin{array}{c}173.98^{\mathrm{bB}} \\
(9.28)\end{array}$ & $\begin{array}{c}189.80^{\mathrm{cB}} \\
(11.12)\end{array}$ & $\begin{array}{l}73.36^{\mathrm{E}} \\
(4.53)\end{array}$ & $\begin{array}{c}82.95^{\mathrm{E}} \\
(5.99)\end{array}$ & $\begin{array}{l}20.31 \\
(2.28)\end{array}$ & $\begin{array}{l}26.76^{\mathrm{f}} \\
(2.71)\end{array}$ & $\begin{array}{l}66.87 \\
(2.67)\end{array}$ & $\begin{array}{l}81.60 \\
(2.39)\end{array}$ & $\begin{array}{c}3.98 \\
(0.79)\end{array}$ & $\begin{array}{c}6.20 \\
(1.37)\end{array}$ & $\begin{array}{l}60.4^{\mathrm{G}} \\
(4.80)\end{array}$ & $\begin{array}{c}65.7^{\mathrm{G}} \\
(3.80)\end{array}$ \\
\hline Group 3 & $\begin{array}{c}218.62^{\mathrm{aC}} \\
(17.72)\end{array}$ & $\begin{array}{c}227.65^{\mathrm{dC}} \\
(18.11)\end{array}$ & $\begin{array}{l}137.38^{\mathrm{e}} \\
(20.30)\end{array}$ & $\begin{array}{l}169.53 \\
(18.31)\end{array}$ & $\begin{array}{l}31.11 \\
(2.43)\end{array}$ & $\begin{array}{l}37.21 \\
(2.08)\end{array}$ & $\begin{array}{l}28.32 \\
(2.32)\end{array}$ & $\begin{array}{l}35.70 \\
(1.86)\end{array}$ & $\begin{array}{c}7.50^{\mathrm{g}} \\
(1.37)\end{array}$ & $\begin{array}{l}10.30^{\mathrm{h}} \\
(1.16)\end{array}$ & $\begin{array}{c}113.6^{\mathrm{iH}} \\
(8.40)\end{array}$ & $\begin{array}{c}118.6^{\mathrm{jH}} \\
(3.60)\end{array}$ \\
\hline Group 4 & $\begin{array}{c}211.27^{\mathrm{aD}} \\
(16.45)\end{array}$ & $\begin{array}{c}228.98^{\mathrm{dD}} \\
(11.26)\end{array}$ & $\begin{array}{l}145.94^{\mathrm{e}} \\
(11.20)\end{array}$ & $\begin{array}{c}200.49 \\
(5.16)\end{array}$ & $\begin{array}{l}24.52 \\
(3.96)\end{array}$ & $\begin{array}{l}28.53^{\mathrm{f}} \\
(1.49)\end{array}$ & $\begin{array}{l}19.19 \\
(2.54)\end{array}$ & $\begin{array}{l}25.49 \\
(2.19)\end{array}$ & $\begin{array}{l}7.55^{\mathrm{g}} \\
(3.52)\end{array}$ & $\begin{array}{l}11.06^{\mathrm{h}} \\
(2.61)\end{array}$ & $\begin{array}{l}101.6^{\mathrm{i}} \\
(14.5)\end{array}$ & $\begin{array}{l}116.9^{\mathrm{j}} \\
(15.2)\end{array}$ \\
\hline
\end{tabular}

Within the same row at each ion, means with the same large superscript letter are not statistically different ( $p>0.01)$. Within the same column, means with the same small superscript are not statistically different $(p>0.01)$.

\section{DISCUSSION}

Previous studies have reported that failure of direct pulp capping treatment is related to bacterial leakage $e^{2,3,15,19)}$. Previous animal studies carried out in our laboratory showed that dentin bridge formation was induced by direct pulp capping with adhesive resin systems after appropriate treatment of the exposed pulp surface ${ }^{15-18)}$. It was also suggested that control of bleeding and exudation from the exposed pulp was important for the success of direct pulp capping ${ }^{20,21)}$. Long-term observation in these studies showed that the dentin bridge formed with resin adhesive system was equal in quantity but slower than that formed with calcium hydroxide. In order to resolve this, an experimental adhesive system containing a dentin-promoting agent, such as $\mathrm{pA} / \mathrm{pB}$ which is a synthetic peptide derived from dentin matrix protein 1 $(\mathrm{DMP} 1)^{17)}$, and calcium phosphate-promoting agent ${ }^{18)}$, such as hydroxyapatite and brushite, was developed for direct pulp capping. The results of these studies showed that direct pulp capping with the experimental adhesive system promoted dentin bridge formation more than that with calcium hydroxide. Thus, these previous studies suggested that an adhesive resin containing a dentinpromoting agent may be used for direct pulp capping. Therefore, we developed a new, all-in-one adhesive containing S-PRG filler for direct pulp capping.

The ions released from the S-PRG filler are trace elements already present in the body. Owing to their outstanding bioactive properties, S-PRG fillers have been used in dental materials such as composite resins ${ }^{24)}$, adhesive resins ${ }^{22)}$, tooth coating material $\mathrm{s}^{27)}$, and fissure sealants ${ }^{25)}$. Several recent studies have shown that S-PRG fillers effectively allow tooth structure strengthening ${ }^{22,28)}$ and acid-resistant layer formation ${ }^{27)}$. The release of various ions, such as $\mathrm{Al}, \mathrm{B}, \mathrm{F}, \mathrm{Na}, \mathrm{Si}$, and $\mathrm{Sr}$, is effective in the prevention of dental caries and periodontal disease. Sr and B ions, in particular, promote osteogenesis and calcification and have been used as osteoporosis treatment drugs and preservatives in eye drops ${ }^{36,39)}$. The biocompatibility of the six ions released from S-PRG filler has been reported previously ${ }^{40)}$. Ito et al. evaluated the cytotoxicity of ions released from the S-PRG filler using HeLa cell culture solution ${ }^{40)}$ and found that the ratio of cell viability in the culture solution with maximum concentration of S-PRG fillers $(10 \mathrm{mg} / \mathrm{mL})$ to that in the control (serum-free medium) was approximately $80 \%$. Generally, it has been proposed that a material cytotoxicity is positive if the cell viability exhibited greater than a $50 \%$ decrease compared to the control $^{41)}$. Therefore, this study suggested that S-PRG fillers may not be cytotoxic. Moreover, the concentration of various ions released from the experimental adhesives appeared to decrease when applied as a direct pulp capping material.

Several studies have reported that uncured resin monomers such as HEMA, Bis-GMA and TEGDMA showed cytotoxicity against mammalian cell ${ }^{42-44)}$. The experimental bonding agents used in the present study contained 2-HEMA and Bis-GMA. However, the results of the present study showed that the experimental adhesive systems for direct pulp capping did not affect the healing process of exposed rat pulp. Other studies have reported that cured bonding resin showed no cytotoxicity against mammalian cells ${ }^{45,46)}$. Therefore, it could be speculated that 2-HEMA and Bis-GMA contained the experimental bonding resins showed almost no cytotoxicity for the pulp tissue because they were photo-polymerized immediately after applied on the exposed rat pulp.

After 14 days, initial or partial dentin bridge formation was observed in the specimens of groups 2, 3 , 4, and control, while no dentin bridge formation was observed in the specimens of group 1, which contained no S-PRG filler. After 28 days, almost all specimens of groups 2, 3, 4, and control exhibited partial or complete dentin bridge formation, while one specimen in group 1 still did not show formation of dentin bridge. Group 2, 
in particular, exhibited better dentin bridge formation compared to the other experimental groups, and no significant differences in the degree of dentin bridge formation were observed between this group and the control after 14 and 28 days post-surgery. Based on these results, the experimentally developed adhesive system containing S-PRG filler, particularly at a concentration of $13 \mathrm{wt} \%$, appears to be effective in direct pulp capping.

Our results also showed that the amount of ions released from the experimental adhesives, and the amount of $\mathrm{Si}$ ion released from the experimental adhesive used in group 2 was significantly greater than that of the other groups. Several studies have reported that $\mathrm{Si}$ ion is fairly effective in remineralization of the dentin matrix ${ }^{31}$. Therefore, it is speculated that abundant release of Si ion may have contributed to the significant dentin bridge formation in group 2 . We also observed slight release of Sr ions from the experimental adhesive used in group 2. It has been confirmed that $\mathrm{Sr}$ ions are effective in inducing calcification during bone formation ${ }^{33-36)}$. In addition, Ammann reported that $\mathrm{Sr}$ ions also promoted differentiation from pre-osteoblasts into osteoblasts and controlled the action of osteoclasts, thus promoting bone formation ${ }^{37}$. Sr hydroxyapatite has been previously used as a dental implant ${ }^{47}$. Therefore, $\mathrm{Sr}$ ions released from the adhesive may also have contributed to dentin bridge formation in group 2 . B ion is associated with brain function, immune regulation, and bone and hormone metabolism ${ }^{38}$. Taşl et al. investigated the cell viability of hTGSCs (human tooth germ stem cell) incubated with different concentrations of NAB (Na pentaborate pentahydrate) using the MTS assay ${ }^{48}$. Their results showed that the cell viability of hTGSCs was increased at low concentrations (10 and $20 \mu \mathrm{g} / \mathrm{mL}$ ) of NAB, but cytotoxicity for hTGSCs was recognized at high concentrations of NAB. They also reported that strong immune-staining for DMP1, COL1A, and Amelogenin was observed in the NAB-treated group. This result suggested that hTGSCs differentiated into odontoblastlike cells due to the bone metabolism function of $\mathrm{B}$ ions released from NAB. Thus, the effect of $\mathrm{B}$ ion on the cell activity is dependent on ion concentration. In this study, the amount of $\mathrm{B}$ ion released from the adhesives used in groups 3 and 4 was approximately twice that released from the adhesive used in group 2. Therefore, it is speculated that the dentin bridge formation may have been affected by the greater amount of $\mathrm{B}$ ions released from the adhesive in groups 3 and 4. On the other hand, the adhesive used in group 2 may release amounts of $\mathrm{B}$ ion that are suitable for promoting differentiation of hTGSCs. The content of S-PRG filler in the experimental adhesive is not proportional to the amount of dentin formed. Assuming that the dental pulp stem cells differentiate into odontoblasts upon stimulation by various released ion, an optimal amount of ions can exist.

The advantages of using adhesive resin system for direct pulp capping include protection of the dentin and pulp from bacterial infection and restoration of the cavity simultaneously. We have previously reported the effects of direct pulp capping using resin adhesive system on wound healing of exposed pulp tissue ${ }^{15-18)}$. Our previous study examined the effects of the experimental twostep self-etch adhesive system containing DMP1 (pA/ $\mathrm{pB})$ as a primer and the powder of calcium phosphate as a bonding agent on direct pulp capping ${ }^{17}$. This study showed that a dentin bridge with a multi-layer structure was formed in the deeper portions of the exposed pulp tissue. The multi-layer structure of the dentin bridge was supposedly generated by diffusion of the primer and bonding agent containing dentin-promoting agents into the pulp tissue. The present study also showed that reparative dentin was formed in the middle and deep portions of pulpal tissues in some specimens. The experimental adhesive systems used in the present study contain 2-hydroxyethyl methacrylate (HEMA), which is a hydrophilic monomer. Therefore, the composition of the adhesives containing S-PRG filler may diffuse from the exposed pulp surface into the deeper portion of the pulp tissue. Furthermore, dentin chips were observed in the pulp tissues of some specimens. Generally, it has been advocated that these dentin chips should be completely removed before placement of the capping agent as they may include bacteria ${ }^{49}$. On the other hand, it has been suggested that leaving these dentin chips in the pulp tissue may induce reparative dentin formation ${ }^{50)}$. In the present study, dentin chips present in the pulp tissue seemed to influence reparative dentin formation but did not cause pulp inflammation. These findings suggest that foreign substances such as fillers or dentin chips may serve as a scaffold for dentin formation.

Silica fillers were observed in the pulp tissue of some specimens of the experimental adhesive groups. Many silica fillers were observed in the pulp tissue of group 1, in particular. The mean particle sizes of the S-PRG fillers and silica fillers were 3 and $1 \mu \mathrm{m}$, respectively. Silica fillers may easily invade the pulp tissue with dentin chips due to its small size compared to the S-PRG fillers. Furthermore, it is also speculated that air-blowing may assist invasion of silica fillers into the pulp tissue. Observation at high magnification showed that the invaded fillers were phagocytosed by fibroblasts. These cells would undergo fibrosis in the long-term. Inoue and Miyakoshi clearly indicated that 4-META monomer penetrated into the soft tissue at a depth of $200 \mu \mathrm{m}$ and formed a hybrid layer, and the micro-granules containing 4-META resin and butanol were also detected around the hybrid layer ${ }^{51)}$. This layer consisted of a soft tissue and a resin monomer causing migration of macrophages ${ }^{51}$. In the present study, no macrophages were observed around the involved fillers. Several studies focusing on direct pulp capping using adhesive resins have reported slight or moderate inflammation in the pulp tissue for short periods ${ }^{15,18,51)}$. Diffusion of unpolymerized monomer into the pulp tissue may cause continuation of mild chronic inflammation. However, the present study showed no inflammatory changes in the pulp tissue of any experimental groups.

In vitro studies were also carried out to examine 
the amount of released ions and the acid buffering capacity of the experimental adhesives contained S-PRG filler. The results showed release of various ions in all experimental materials. The acid buffering capacity of groups 2, 3, and 4 was higher than that of group 1 , and the $\mathrm{pH}$ value of groups 2,3 , and 4 became neutral after $24 \mathrm{~h}$. Therefore, it was suggested that the amount of S-PRG filler could be correlated with the acid buffer capacity. These results were in agreement with earlier studies $^{28}$. Moreover, increasing amounts of S-PRG filler and storage terms resulted in larger amounts of released $\mathrm{B}, \mathrm{Sr}, \mathrm{F}$, and $\mathrm{Na}$ ions. Other studies have also concluded that S-PRG filler content affected the amount of released ion $^{23,52)}$. Han and Okiji indicated that release of B, F, and $\mathrm{Al}$ ions gradually decreased with the passage of time ${ }^{26}$.

Based on the results of the present study, the null hypothesis that the S-PRG filler content in the experimental adhesives would not affect pulpal healing and tertiary dentin formation in the exposed rat pulp was not rejected. The dentin bridge formation was dependent on the amount of S-PRG fillers, and the experimental adhesive containing $13 \mathrm{wt} \% \mathrm{~S}$-PRG fillers exhibited the best dentin bridge formation among the experimental adhesives. However, there were some limitations with this adhesive system, such as penetration of resin monomer and invasion of silica fillers into the exposed pulp surface. In the future, it will be necessary to develop a low viscosity self-adhesive resin system for direct pulp capping in order to control invasion of the material contents into the exposed pulp surface.

\section{CONCLUSION}

All experimental adhesives containing S-PRG fillers developed for direct pulp capping showed no pulpal inflammation. After 14 days post-surgery, the experimental adhesives containing S-PRG fillers and the control group formed reparative dentin around the exposed pulp; however, most specimens did not form dentin bridge. After 28 days post-surgery, the respective experimental adhesives containing S-PRG fillers of 13 and $27 \mathrm{wt} \%$ formed dentin bridge almost equal to the control.

\section{ACKNOWLEDGMENTS}

This study was supported by a Grant-in-Aid for Scientific Research (No.25462971) from the Japan Society for the Promotion of Science (JSPS). The authors thank Shofu for the experimental adhesive resin systems and other materials they generously provided.

\section{CONFLICT OF INTEREST}

The authors declare that they have no competing interests.

\section{REFERENCES}

1) Schröder U. Effects of calcium hydroxide-containing pulp- capping agents on pulp cell migration, proliferation, and differentiation. J Dent Res 1985; 64: 541-548.

2) Cox CF, Suzuki S. Re-evaluating pulp protection: calcium hydroxide liners vs. cohesive hybridization. J Am Dent Assoc 1994; 125: 823-831.

3) Cox CF, Subay RK, Ostro E, Suzuki S, Suzuki SH. Tunnel defects in dentin bridges: Their formation following direct pulp capping. Oper Dent 1996; 21: 4-11.

4) Fujitani M, Shibata S, Van Meerbeek B, Yoshida Y, Shintani H. Direct adhesive pulp capping: pulpal healing and ultramorphology of the resin-pulp interface. Am J Dent 2002; 15: 395-402.

5) Kitasako Y, Inokoshi S, Fujitani M, Otsuki M, Tagami J. Short-term reaction of exposed monkey pulp beneath adhesive resins. Oper Dent 1998; 23: 308-317.

6) Akimoto N, Momoi Y, Kohno A, Suzuki S, Otsuki M, Suzuki S, Cox CF. Biocompatibility of Clearfil Liner Bond 2 and Clearfil AP-X system on non-exposed and exposed primate teeth. Quintessence Int 1998; 29: 177-188.

7) Swarup SJ, Rao A, Boaz K, Srikant N, Shenoy R. Pulpal response to nano hydroxyapatite, mineral trioxide aggregate and calcium hydroxide when used as a direct pulp capping agent: an in vivo study. J Clin Pediatr Dent 2014; 38: 201206.

8) Chohayeb AA, Adrian JC, Salamat K. Pulpal response to tricalcium phosphate as a capping agent. Oral Surg Oral Med Oral Pathol 1991; 71: 343-345.

9) Al-Hezaimi K, Al-Tayar BA, Bajuaifer YS, Salameh Z, AlFouzan K, Tay FR. A hybrid approach to direct pulp capping by using emdogain with a capping material. J Endod 2011; 37: 667-672.

10) Kuratate M, Yoshiba K, Shigetani Y, Yoshiba N, Ohshima H, Okiji T. Immunohistochemical analysis of nestin, osteopontin, and proliferating cells in the reparative process of exposed dental pulp capped with mineral trioxide aggregate. J Endod 2008; 34:970-974.

11) de Souza Costa CA, Lopes do Nascimento AB, Teixeira HM, Fontana UF. Response of human pulps capped with a selfetching adhesive system. Dent Mater 2001; 17: 230-240.

12) Pereira JC, Segala AD, Costa CA. Human pulpal response to direct pulp capping with an adhesive system. Am J Dent 2000; 13: 139-147.

13) Koliniotou-Koumpia E, Tziafas D. Pulpal responses following direct pulp capping of healthy dog teeth with dentine adhesive systems. J Dent 2005; 33: 639-647.

14) Silva GAB, Lanza LD, Lopes-Júnior N, Moreira A, Alves JB. Direct pulp capping with a dentin bonding system in human teeth: a clinical and histological evaluation. Oper Dent 2006; 31: 297-307.

15) Medina VO III, Shinkai K, Shirono M, Tanaka N, Katoh Y. Histopathologic study on pulp response to single-bottle and selfetching adhesive systems. Oper Dent 2002; 27: 330-342.

16) Suzuki M, Katsumi A, Watanabe R, Shirono M, Katoh Y. Effects of an experimentally developed adhesive resin system and $\mathrm{CO} 2$ laser irradiation on direct pulp capping. Oper Dent 2005; 30: 702-718.

17) Taira Y, Shinkai K, Suzuki M, Kato C, Katoh Y. Direct pulp capping effect with experimentally developed adhesive resin systems containing reparative dentin-promoting agents on rat pulp: mixed amounts of additives and their effect on wound healing. Odontology 2011; 99: 135-147.

18) Kato C, Suzuki M, Shinkai K, Katoh Y. Histopathological and immunohistochemical study on the effects of a direct pulp capping experimentally developed adhesive resin system containing reparative dentin-promoting agents. Dent Mater J 2011; 30: 583-597.

19) Cui C, Zhou X, Chen X, Fan M, Bian Z, Chen Z. The adverse effect of self-etching adhesive systems on dental pulp after direct pulp capping. Quintessence Int 2009; 40: 26-34. 
20) Kitasako Y, Inokoshi S, Tagami J. Effects of direct resin pulp capping techniques on short-term response of mechanically exposed pulps. J Dent 1999; 27: 257-263.

21) Hafez AA, Cox CF, Tarim B, Otsuki M, Akimoto N. An in vivo evaluation of hemorrhage control using sodium hypochlorite and direct capping with a one- or two-component adhesive system in exposed nonhuman primate pulps. Quintessence Int 2002; 33: 261-272.

22) Han L, Okamoto A, Fukushima M, Okiji T. Evaluation of a new fluoride-releasing one-step adhesive. Dent Mater J 2006; 25: 509-515.

23) Fujimoto Y, Iwasa M, Murayama R, Miyazaki M, Nagafuji A, Nakatsuka T. Detection of ions released from S-PRG fillers and their modulation effect. Dent Mater J 2010; 29: 392-397.

24) Ito S, Iijima M, Hashimoto M, Tsukamoto N, Mizoguchi I, Saito T. Effects of surface pre-reacted glass-ionomer fillers on mineral induction by phosphoprotein. J Dent 2011; 39: 7279.

25) Shimazu K, Ogata K, Karibe H. Evaluation of the ionreleasing and recharging abilities of a resin-based fissure sealant containing S-PRG filler. Dent Mater J 2011; 30: 923927.

26) Han L, Okiji T. Evaluation of the ions release/incorporation of the prototype S-PRG filler-containing endodontic sealer. Dent Mater J 2011; 30: 898-903.

27) Ma S, Imazato S, Chen JH, Mayanagi G, Takahashi N, Ishimoto T, Nakano T. Effects of a coating resin containing S-PRG filler to prevent demineralization of root surfaces. Dent Mater J 2012; 31: 909-915.

28) Kaga M, Kakuda S, Ida Y, Toshima H, Hashimoto M, Endo K, Sano H. Inhibition of enamel demineralization by buffering effect of S-PRG filler-containing dental sealant. Eur J Oral Sci 2014; 122: 78-83.

29) Iijima Y, Koulourides T. Fluoride incorporation into and retention in remineralized enamel. J Dent Res 1989; 68: 1289-1292.

30) Itota T, Nakabo S, Iwai Y, Konishi N, Nagamine M, Torii Y. Inhibition of artificial secondary caries by fluoride-releasing adhesives on root dentin. J Oral Rehabil 2002; 29: 523-527.

31) Saito T, Toyooka H, Ito S, Crenshaw MA. In vitro study of remineralization of dentin: effects of ions on mineral induction by decalcified dentin matrix. Caries Res 2003; 37: 445-449.

32) Itthagarun A, Tay FR, Pashley DH, Wefel JS, García-Godoy F, Wei SH. Single-step, self-etch adhesives behave as permeable membranes after polymerization. Part III. Evidence from fluid conductance and artificial caries inhibition. Am J Dent 2004; 17: 394-400.

33) Boivin G, Deloffre P, Perrat B, Panczer G, Boudeulle M, Mauras Y, Allain P, Tsouderos Y, Meunier PJ. Strontium distribution and interactions with bone mineral in monkey iliac bone after strontium salt (S 12911) administration. J Bone Miner Res 1996; 11: 1302-1311.

34) Grynpas MD, Hamilton E, Cheung R, Tsouderos Y, Deloffre P, Hott M, Marie PJ. Strontium increases vertebral bone volume in rats at a low dose that does not induce detectable mineralization defect. Bone 1996; 18: 253-259.

35) Christoffersen J, Christoffersen MR, Kolthoff N, Bärenholdt O. Effects of strontium ions on growth and dissolution of hydroxyapatite and on bone mineral detection. Bone 1997; 20: $47-54$.
36) Saint-Jean SJ, Camiré CL, Nevsten P, Hansen S, Ginebra MP. Study of the reactivity and in vitro bioactivity of Srsubstituted alpha-TCP cements. J Mater Sci Mater Med 2005; 16: 993-1001.

37) Ammann P. Strontium ranelate: A physiological approach for an improved bone quality. Bone 2006; 38: 15-18.

38) Nielsen FH, Meacham SL. Growing evidence for human health benefits of boron. J Evid Based Complementary Altern Med 2011; 16: 169-180.

39) Ayaki M, Iwasawa A. Cell viability of four corneoconjunctival cell lines exposed to five preservatives and a surfactant used for infection control in eyedrops. Biocontrol Sci 2011; 16: 117121.

40) Ito N, Morikawa T, Kotake H, Hotta M. Cytotoxicity of HeLa cells cultured on S-PRG filler. Jpn J Conserv Dent 2011; 54: 26-32.

41) Takimoto T, Takeda S. The effects of cell density, exposure time and assay method on evaluation of cytotoxicity of chemicals. J J Dent Mater 1991; 10: 431-442.

42) Hanks CT, Strawn SE, Wataha JC, Craig RG. Cytotoxic effects of resin components on cultured mammalian fibroblasts. J Dent Res 1991; 70: 1450-1455.

43) Ratanasathien S, Wataha JC, Hanks CT, Dennison JB. Cytotoxic interactive effects of dentin bonding components on mouse fibroblasts. J Dent Res 1995; 74: 1602-1606.

44) Kehe K, Reichl FX, Durner J, Walther U, Hickel R, Forth W. Cytotoxicity of dental composite components and mercury compounds in pulmonary cells. Biomaterials 2001; 22: 317322.

45) Kaga M, Noda M, Ferracane JL, Nakamura W, Oguchi H, Sano H. The in vitro cytotoxicity of eluates from dentin bonding resins and their effect on tyrosine phosphorylation of L929 cells. Dent Mater 2001; 17: 333-339.

46) Banava S, Najibfard K, Garcia-Godoy F, Saghiri MA, Ghahremani MH, Ostad N. Impact of dilution and polymerization on cytotoxicity of dentin adhesives to human gingival fibroblasts: early exposure time. J Dent Res Dent Clin Dent Prospects 2015; 9: 151-158.

47) Hao J, Chou J, Kuroda S, Otsuka M, Kasugai S, Lang NP. Strontium hydroxyapatite in situ gel-forming system - a new approach for minimally invasive bone augmentation. Clin Oral Implants Res 2015; 26: 581-585.

48) Taşlı PN, Doğan A, Demirci S, Şahin F. Boron enhances odontogenic and osteogenic differentiation of human tooth germ stem cells (hTGSCs) in vitro. Biol Trace Elem Res 2013; 153: 419-427

49) Holland R, de Souza V, de Mello W, Nery MJ, Bernabé PF, Otoboni Filho JA. Permeability of the hard tissue bridge formed after pulpotomy with calcium hydroxide: a histological study. J Am Dent Assoc 1979; 99: 472-475.

50) Katoh Y. Clinico-pathological study on pulp-irritation of adhesive resinous material, report 1-histopathological change of the pulp tissue in direct capping. Adhes Dent 1993; 11: 119211.

51) Inoue T, Miyakoshi S. Influence of 4-META/MMA-TBB resin on pulp tissue. Adhes Dent 2014; 32: 36-62.

52) Kamijo K, Mukai Y, Tominaga T, Iwaya I, Fujino F, Hirata Y, Teranaka T. Fluoride release and recharge characteristics of denture base resins containing surface pre-reacted glassionomer filler. Dent Mater J 2009; 28: 227-233. 